\title{
Challenges of today: at one side employment important for quality of life and to avoid depression; at the other side a worldwide economic crisis
}

\author{
JJ Wyndaele \\ Antwerp University Hospital, Antwerp, Belgium \\ E-mail: wyndaelejj@skynet.be
}

Dear Spinal Cord reader,

We read it or hear it stated every day: the economic crisis and global world changes have not found a new equilibrium as yet. This hurts everyone and also those individuals who suffer a SC lesion. The solution will not be by one person but by general effort, strong belief in the future, solidarity and offer of mutual support. An impressive but feasible program to make a breakthrough.

In this SC issue there are several nice studies and reports.

Krueger et al. developed a conceptual model to explore the relationship between the presence of depressive symptoms and secondary physical complications such as pressure ulcers, urinary tract infections, and autonomic dysreflexia in SCI.

QoL differences between 6 countries (Australia, Brazil, Canada, Israel, South Africa, and the United States) have been found by Geyh et al, that could not be explained by differences in demographic and lesion characteristics. Results point to the relevance of reintegration of people with SCI into the workforce.

Krebs and Pannek found Solifenacin, an antimuscarinic drug, to be an effective oral treatment of neurogenic detrusor overactivity after SCI.

Gohbara et al. showed that a selection of urinary management measures for patients with incomplete cervical cord injuries can be performed adequately by considering the severity of paralysis and urinary sensation.

Haas et al. studied the psychological and emotional support provided by a peer support officer and found it highly valued by the participants. The peer support officer's direct experience of living with a spinal injury gave credibility to the practical advice, information and signposting provided; as well as to the empathy demonstrated by them during their involvement with people with SCI and their families. Healthcare professionals appreciated their unique perspective and considered them a valuable member of the multidisciplinary team.

Schuld et al. quantified the effect of formal training in the International Standards for Neurological Classification of Spinal Cord Injury on the classification accuracy and identified the most difficult of its rules.

Wong et al. showed that endurance training is related to increased small artery compliance in persons with SCI as good as in healthy controls. Endurance training may extenuate the decline in small artery compliance seen after SCI.

De Groot et al. present their investigation on the course of coronary heart disease risk factors (lipid profiles and body mass index (BMI)) in the first five years after discharge of inpatient SCI and the association with physical activity, self-care related to fitness, smoking, alcohol, body mass and low-fat diet.

Sullivan et al. evaluated what is rarely studied: oral health. It appears to be compromised in people with SCI. Identification of modifiable risk factors and prevention is warranted.

Shimizu et al. described communicating by electrolarynx with a blind individual with tetraplegia on artificial ventilation.

Sitting and seating is the subject of the papers by Serra-Anó et al. (Static and dynamic seated-balance) and Taule (factors that predict unsatisfactory seating pressure).

Morris et al. found able-bodied individuals' appraisals of disability after imagined SCI much more negative than the actual appraisals of disability in real SCI individuals. There are many more interesting papers in this issue.

Enjoy reading and do not hesitate to contact us at Spinalcord@uza.be. 